\title{
Education Needs to Support Architecture, Engineering, and Construction Collaboration Using Building Information Modeling
}

\author{
Dilan M. Rostam \\ Department of Architectural Engineering, Faculty of Engineering, Koya University, \\ Kurdistan Region - F.R. Iraq
}

\begin{abstract}
Projects in the construction industry involve multidisciplinary collaboration between the disciplines of architecture, engineering, and construction (AEC), and others. Conventionally, the collaboration between these disciplines relied on the recurrent exchange of relevant drawings and documents. Building information modeling (BIM) as a model-based process has given AEC professionals the tools to more efficiently plan, design, construct, and manage buildings and infrastructure. Yet the AEC industry has been reluctant in fully adopting the BIM as a single standard. This study explores and identifies the bottlenecks in adopting BIM as a single product lifecycle standard in the construction industry and advise on educating new engineers to become the generation to use a virtual collaborative working space covering the entire building lifecycle. Two conducted surveys targeting the AEC academia and industry revealed the needs for multilevel cross-disciplinary interactive collaborative BIM process modeling, and skilled workforce to increase the graduates' marketability and BIM adaptability. It is concluded that the new age collaborative culture requires new generation of AEC players that are enabled to work on a shared virtual product model supported by proactive BIM skills learned through undergraduate programs.
\end{abstract}

Index Terms-Architecture; engineering; and construction collaboration, Building information modeling, Building lifecycle, Education, Integrated design.

\section{INTRODUCTION}

Population is increasingly choosing concentrated urbanism where the demand for various services is growing exponentially to accommodate life. Sustainable cost-effective buildings with longer lifespan are at the center of these services. In response to these, the construction industry has adopted various measures such as rapid industrial building techniques. It is evident that such techniques have been commonly used in manufacturing industries for decades. However, the

ARO-The Scientific Journal of Koya University

Volume VII, No.2 (2019), Article ID: ARO.10604, 10 pages

DOI: $10.14500 /$ aro. 10604

Received: 29 November 2019; Accepted: 08 December 2019

Regular research paper: Published 20 December 2019

Corresponding author's, e-mail: dilan.rostam@koyauniversity.org Copyright (C) 2019 Dilan M. Rostam. This is an open-access article distributed under the Creative Commons Attribution License. use of industrialized construction is also expanding in the architecture, engineering, and construction (AEC) industry in the form of building information modeling (BIM) to improve planning, design, construction, and collaboration for increased sustainability, lower costs, and broader safety throughout the lifecycle (Dassault Systèmes, 2014).

The US National BIM Standard defines BIM as a "digital representation of physical and functional characteristics of a facility; shared knowledge resource for information about a facility forming a reliable basis for decisions during its life cycle; also defined as existing from earliest conception to demolition" (NBS-US, 2015). A basic idea of BIM is a collaboration by all actors involved in the building process throughout the lifecycle. The information in the BIM can be extracted, updated, or modified to support and reflect the roles of an actor (Bazjanac, 2006). Therefore, BIM is information modeling and information management in a collaborative digital working environment for the AEC building process. BIM creates a product model based on collective efforts of a coordinated working process using responsive technology which makes it a collaborative e-working environment by nature.

Olofsson, et al. (2008) stated that the major key advantages of BIM are that allow the development of detailed information and analysis at a very early stage of the building process to improve decision making and reduce cross-discipline changes. The primary benefits of early stage engagement of BIM that can be identified are "(1) rapid visualization, (2) better decision support upstream in the project development process, (3) rapid and accurate updating of changes, (4) reduction of labor required to establish reliable space programs, (5) increased communication across the life cycle, and (6) increased confidence in completeness of scope" (Manning and Messner, 2008). Their study of using BIM in some projects reveal clear improvement in engineering design quality, in terms of more accurate drawings, and steadily increasing improvement in labor productivity.

It is evident that the AEC industry is highly interdisciplinary by nature that requires multidisciplinary instructions in the design process. Globally, the AEC companies are required to build more rapidly, be sustainable, improve quality, and satisfy the environmental regulations. 
These constraints are currently leading cultural changes in the way, the AEC industry is managing projects (Dassault Systèmes, 2014). BIM is facilitating the core of these cultural changes. However, BIM is not simply a type of software but a process that includes multidisciplinary actors that need to work collaboratively to generate and manage all-inclusive building data. Not only do BIM represents a three-dimensional intelligent model of the building but also "making significant changes in the workflow and project delivery processes" (Hardin, 2009). This brings all previous fragmented subprocesses to one inclusive platform.

Although understanding and mastering the concept of a multidisciplinary virtual working environment based on a single shared product model will by nature bring AEC industry closer than ever, unfortunately today in AEC industry BIM potential is mainly used in isolated design tasks and not for a sustained collaboration process (Thomassen, 2011). This is despite all the increasing usage of digital devices in everyday life, but still the concept of BIM has taken more than 20 years to become the preferred process of the industry in some degree today (Eastman, et al., 2008). Therefore, the focus of this work is to investigate the current views on how BIM can affect the collaborative concept of AEC industry, and in what way the adaptability of the industry toward BIM implementation can be increased.

BIM is not only about how a shared e-working space work technically but also how to act and play a role in such an environment. Therefore, the rules of conduct by actors have direct relation with their level of BIM understanding and skills.

The study aims to explore the collaboration concept of a shared digital model using BIM among AEC professionals through conducting surveys to examine the state of adopting BIM as a collaboration tool mainly in the UK and make recommendations for the best possible path to promote BIM as a single working environment and increase its usability.

\section{Methodology}

This research collects data from literature to find out current directions in BIM implementations and value for rational collaboration in the construction industry. After the initial studies, further data were collected through two surveys to identify the industry's state of engagement with BIM and their expected collaboration frameworks. They were asked to state their expectations, practical needs and views and the possible impact in collaborative work within the same organization or cross organizations with significant geographic distribution using BIM environment. The second survey dealt with validation of the existing undergraduate programs to understand the future graduates' basic capacity to work in a BIM oriented collaborative working environment as well as culture of BIM concept in education. Findings from the two surveys were used to identify shortcomings in the capacity building for a BIM oriented working environment. This includes BIM and team collaboration within the construction industry context, BIM management, web-based collaborative solutions, and cloud computing.

\section{INDUSTRY's COLlaboration NeEDS}

BIM is one of the most promising developments in the AEC industries (Eastman, et al., 2008) where cross multidisciplinary collaboration and exchange of large building data are required. Conventionally, the means for collaboration sense between actors have been based on the repeated exchange of two-dimensional drawings and documents in form of hard or electronic copies. The long experience of the AEC industry shows that cross-disciplines engagements in preparing of model details and workflow at an early stage of a project are vital to successful design collaboration. However, during the past decade, the global adoption of computer-aided design (CAD) tools has paved the way for BIM as reliable alternative for early interaction acts in AEC projects processing (Singh, et al., 2011).

Singh, et al. (2011) showed that the expectations of BIM as a tool for collaboration are directly dependent on the construction industry's disciplines. For design disciplines, $\mathrm{BIM}$ is more an extension to CAD, whereas for others such as contractors and project managers, BIM is an intelligent shared document management system that can quickly take off data from CAD packages directly. However, the visualization of the shared product model is an import requirement across all disciplines, which today makes BIM an important factor in collaboration across the AEC industry.

AEC industries struggle to more productive collaboration is not only about the technological requirements for multidisciplinary interactions but also replacing the long tradition of acting task-based working environment with a new responsive culture of shared electronic product model that brings everyone together with provide adequate support for their particular discipline. Therefore, the success of BIMbased collaboration is directly depending on the adaptation and mindset ability of working in such a shared environment.

In a series of workshops and interviews that were carried out by Bhargav and Lauri (2009) with various building project actors, the following requirements were identified that provide basic insight into what collaborative act means to people involved and how it can be realized. They would like to be able to share ideas in real time, be able to collaborate on a common platform, be able to discuss an idea in a simple way, post any sort of related documents, to search within solutions and previous exchange/documents, ease of use and intuitive interface. These requirements are summarizing the human understanding of the chain of analyzing, processing and decision makings of data in relation to collaboration on a common product model.

Azhar (2011) reveals analyses of the status of BIM in the AEC survey market in the USA and mentions that the AEC industry still relies very much on traditional drawings and practices for processing their projects. At the same time, AEC professionals are realizing the power of BIM for more efficient and intelligent modeling and meaningful collaboration. Most of the companies using BIM reported in strong favor of this technology. The survey findings indicate that users want a BIM application that not only benefits from the powerful documentation and visualization capabilities 
of a CAD platform but also supports inclusive design and collaboration acts. Consequently, BIM as technology is improving, and solutions in the market are continuing to evolve as they respond to the user's specific needs (Azhar, 2011).

Many studies are focusing on single disciple such as Shin (2017) and Kaner (2008) also confirmed that BIM and its impact in contributing to collaborative product design from various perspectives. They studied the impact of BIM on the structural engineering collaboration process and concluded that the work efficiency and the quality of the buildings improved when BIM collaboration was used for structural information.

Eastman, et al. (2008) introduces a comparison model based on wide studies on real AEC project to illustrate the importance of the data losses during the lifecycle of a building in a traditional paper-based process against a collaborative BIM-based delivery process (Fig. 1).

It is clear that the AEC industry as a whole has come to terms with the impact of BIM and the necessity for closer interactions in future product model processing. This product model will become the hub for interaction between all actors from concept to end of the lifespan. In other words an object-based product modeling with detailed intelligent building data. Therefore, governments around the world are setting out rules and regulations to lead the industry to full BIM adoption path to single AEC product modeling standard in targeted implementation BIM levels $0,1,2$, and 3 (Fig. 2).

The UK BIM strategy was reinforced in 2011, which directed the construction industry to make BIM mandatory on all public projects by April 2016 to at least "level 2" BIM maturity. The concept of "BIM levels" (and "BIM level 2 compliance") has become the "accepted" definition by industry, by seeing the adoption process as the next steps in a process that has taken the AEC industry from the drawing boards to the CAD and, finally, into the shared product model in the digital age (NBS, 2014).

According to NBS, 2014, BIM levels are a government adoption process from a level to the next level for moving the construction industry to "full" collaborative working in a progressive, distinct, and recognizable way. Mills (2015) states these levels run from 0 to 3 are used as reference measures of BIM maturity.

However, the current collective historical experiences and requirements in AEC industry have brought to live the concept of level 3 BIM which represents a full collaboration between all disciplines on a shared product model in a common working space. All parties can access and modify the shared model with anticipated rights and dependencies that eliminates the risk for conflicting information, which allow collaboration between actors (Khanzode et al., 2008).

BIM level 3 extended collaboration and product lifecycle management tools can minimize waste and costs, improve sustainability, and lower the overall building process cost as well as introducing a new culture of AEC collaboration.

\section{SuRvey AND ANALYSis on AEC COLlaboration}

The BS 1192:2007 and the complementary document PAS BS 1192-2:2013 state that the collaboration between the participants in construction projects is vital to the efficient delivery of product models. The guidelines provide instruction to the AEC industry which is increasingly working in new collaborative environments to achieve higher standards of quality and extending reuse of existing knowledge and experience (BS 1192, 2007).

Currently, in the majority of real-life AEC projects, the collaboration act taking place as the user of a tool in one discipline will import IFC models from other disciplines to their local computer application. If there is an problem related to one of the imported models, the best response by

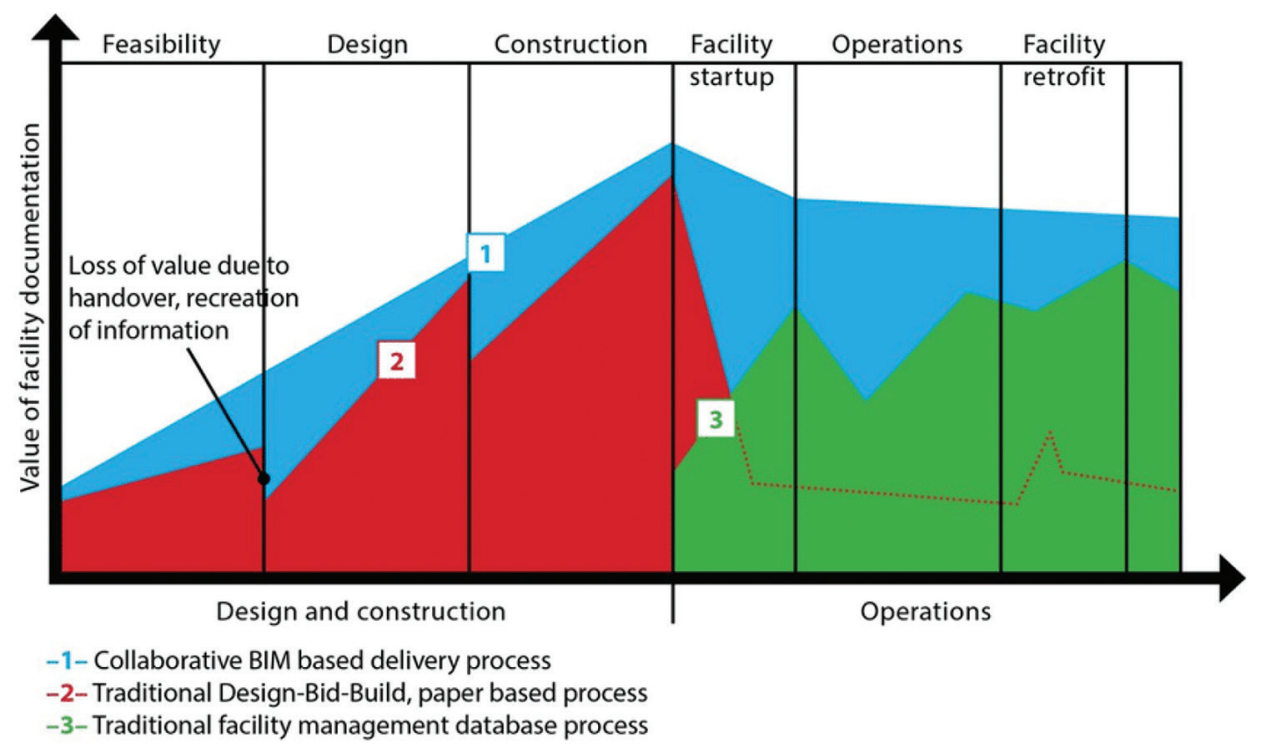

Fig. 1. Data integrity (Eastman, et al., 2008): Graphic presentation of the data losses during the lifetime of a building in a traditional paper-based process versus a collaborative building information modeling-based delivery process. 


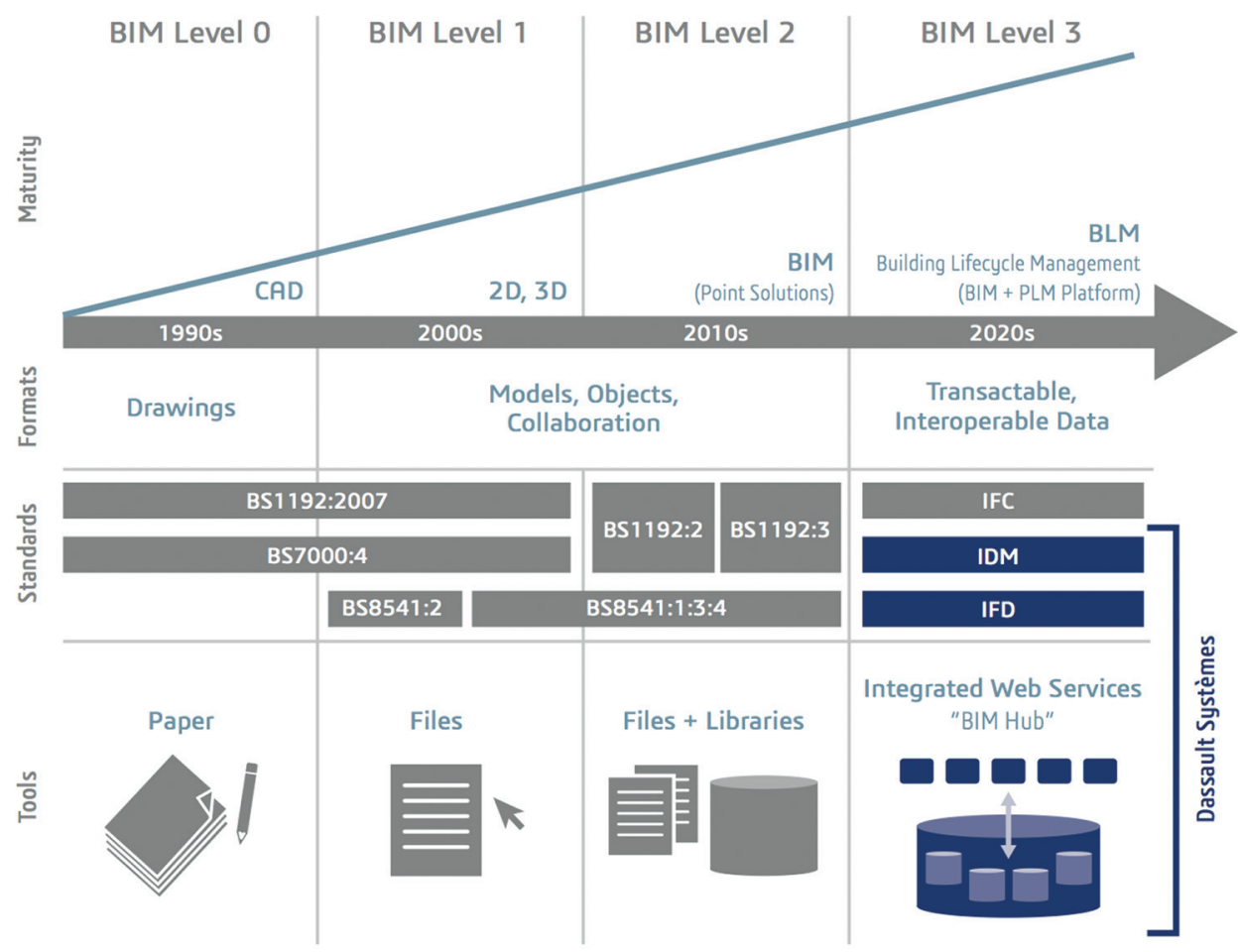

Fig. 2. An updated building information modeling maturity model from computer-aided design to building lifecycle management (Dassault Systèmes, 2014).

the user is to report that problem so they can be resolved in the original BIM authoring application from where this model originated (Stangeland, 2011).

Azhar (2011) carried out a survey to understand the current and future trends in collaboration, from a practical view. He explained that the study disclosed a number of concerns in today's collaboration conduct which circulated toward "process, technology, and people related problems" (Azhar, 2011). However, it is evident that the diversity of existing collaboration systems, compatibility problems, "training and learning curve, controlling the BIM, change and version management in models, ownership and responsibility of model data, intellectual property rights, reliability of model contents and the volatile nature of models, and uncertainty of the BIM market" obstruct the scope of BIM collaboration (Shafiq, et al., 2012).

This study carried out two parallel surveys to evaluate the current position in relation to the concept of BIM implementation and collaboration in the AEC industry and academic institutes. The study managed to collect 280 responses from academics and 89 from the industry. The survey was a parallel study between the UK and Kurdistan Region of Iraq (KRI) where only 39 academics responded and no companies responded to the survey. However, over $70 \%$ of responders to both surveys believed that collaboration is only meaningful when a common platform for real interaction exists (Fig. 3). At the same time, $85 \%$ said there should be a motivation in place for adjoining new technology that supports active collaboration as for the moment; the trends are very slow and not preferable. Many identified $\mathrm{BIM}$ as an emerging process empowering more meaningful collaboration in the right direction.
Over $72 \%$ of the participants agreed that collaboration through the BIM platform increased their overall productivity and quality of this project outcome. This understanding indicated to be directly related to simple, available, and sensible communication tools that eliminated the redoing and recurrent tasks. Many respondents believed that using BIM brought changes to their normal routine work and they acted differently accordingly to fit within the circumstances. However, the AEC industry still faces many challenges to adopt BIM strategies as a single alternative for project processing. The majority of the respondents indicated that lack of knowledge and clarity, leadership strategies, motivation, and lack of training are among clear reasons behind the slow adoption of BIM (Fig. 4).

This shows that there are clear indications that the virtual collaborative working environment requires a new type of skills that directing the new generation project processing more effectively. Howard and Björk (2008) also advocate the reforming of the building process rather than focusing on new technological concepts; therefore, with BIM implementation, there is a requirement to engage a new BIM skilled role within the multidisciplinary team to overview the coordination of the process model. Such perception of the future AEC project processing has led to many new adjustments in how the public sectors are engaging the AEC with their projects. The AEC industry market statistics show that the UK Government's requirements to use BIM in projects have led many organizations adopting BIM for the first time (Eadie, et al., 2015). Therefore, there is a tremendous pressure on the AEC industry to increase their marketability and working 


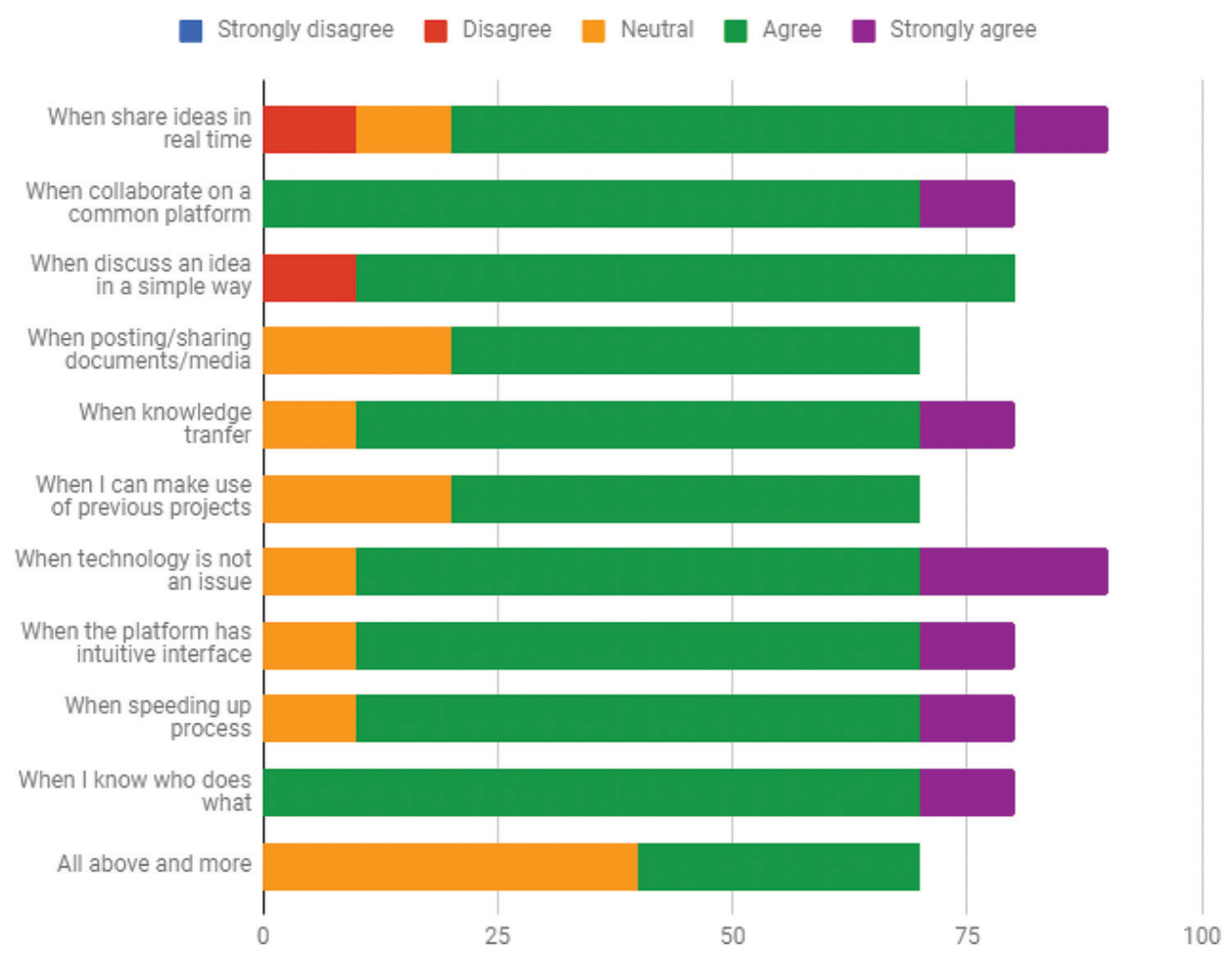

Fig. 3. Majority of respondents believed that a common platform, simplicity, and clarity in actions are empowering meaningful collaboration.

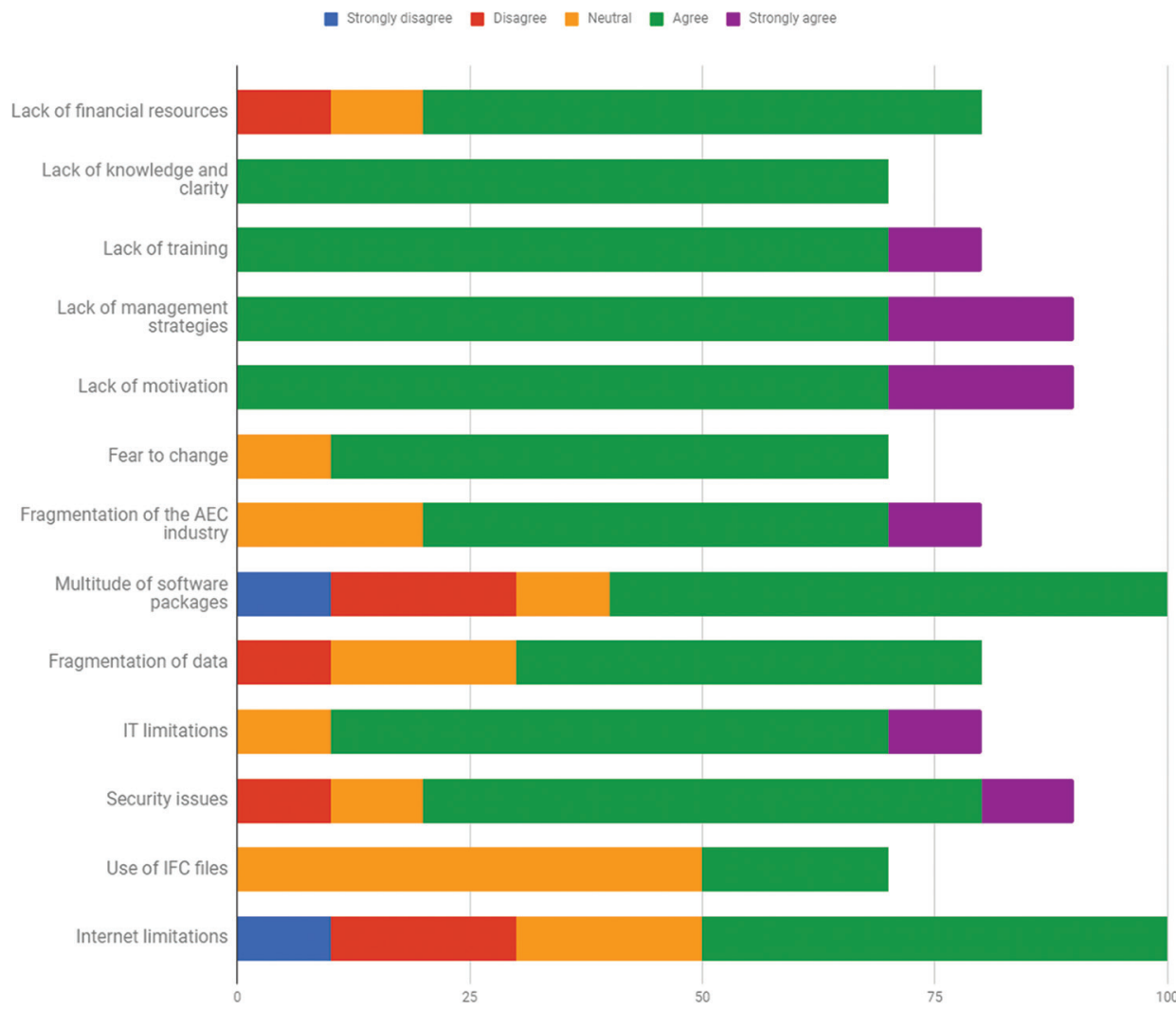

Fig. 4. Architecture, engineering, and construction industry's challenges in adopting clear building information modeling strategies.

fashion by engaging in next BIM level 3 and bring further closer all-inclusive collaboration in shared electronic working space that has positive impacts throughout the lifecycle of the product model.

\section{Shared Product Model on BIM-cloud}

All AEC software vendors have built software to fulfill their specific tasks and talk to a computer but that does not necessarily mean they talk to other actors involved in 
the design process. As a result, the computer and human communication on the same design product is immensely isolated.

The global nature of the AEC industry and the international treaties focusing on the industrial impact on carbon emission levels govern a new concept of industrialization of the AEC industries. To keep a proactive role in this new era, all global players need to get involved and have a common roadmap and be competitive in the wider market with a new strategy.

Evidently the AEC industry tends to realize such strategy by putting a model at the center of the project and creating BIM, which will be possible to engage with deeper information into the cost and constructability. Therefore, connecting actors and technologies used in the instruction will make it possible to work smarter and make better decisions (Autodesk 2015). These trends are clear indications for Software Industry to respond accordingly.

Software vendors specializing in providing tools to the AEC industry led by users' requirements and the rapid advancement of IT are pushing for better marketability in a dynamic ever-changing market. They are looking for easy access scalable products; therefore, the cloud computing has opened a limitless possibility for innovative software. Simply explained, cloud computing is providing computing services such as storage, databases, software, and more using the Internet "the cloud." With the internet accessibility, usage and reliability on the rise it is practical to use a network of geographically distributed servers hosted on the internet to store, manage, and process data, rather than a local server or a desktop computer. This has made cloud computing to a key aspect of realizing BIM level 3 by the future for the industry that allows for real time multidisciplinary remote access collaboration within an ultimate internet browser-based BIM environment.

Singh, et al. (2011) defined a BIM shared working workspace as a collaboration platform that retains a repository of the entire building data. However, he reported many practical problems in using such platforms that have a direct impact on the collaboration process. These shortcomings identified mostly in role dependencies and responsibilities, which are critical to the setup and access to the integrated BIM model as well as interactive help functions within general (Singh, et al., 2011).

There are clear indications of cultural changes in the construction industry, in particular, the concept of collaboration and its impact on budgets and product lifecycle, which are now addressing Singh, et al. (2011) concerns. Currently, many specialized companies are already providing solutions for the entire construction lifecycle inform of collaborative platforms to facilitate the engagement around shared product models and documentations, such as Aconex (aconex.com), TeamBinder (teambinder.com), and Tekla BIMsight and RIB (rib-software.com.au). Company such as Aconex claims that they have processed 16,000 projects of $\$ 1$ trillion in value from 70 countries with greater geographically distributed nature (Aconex, 2017). At the core of services provided by these companies lays the new trend in working with shared BIM and data interoperability that the IFC data set provides. Such a shared product model concept was advised and tested already in small scale by implementation and evaluation of a virtual collaborative design environment system at the University of Nottingham back in 2005 (Roshani and Tizani, 2005).

However, despite the construction industry's recent advances in technology adoption, overall management of those tools remains irregular, and the adaptation process is very slow. According to a recent survey of construction industry professionals and academic community by this study, $72 \%$ said that they have collaborated using BIM in some form in their line of work, but only $55 \%$ has received any sort of BIM training, whereas over $65 \%$ agreed that using BIM has improved company's overall productivity and project outcomes. In comparison between common software programs and cloud-based systems, the responders believed that the current available software caused data duplication problems, redundant activity, and wasted time. Further, $45 \%$ found accessing the latest set of documents and having the most current information to be a challenge. Nonetheless, more than $90 \%$ said that cloud technology is the most practical option for the industry although they showed a moderate level of confidence in BIM level 3 (Fig. 5).

There is a huge difference between the requirements for collaboration within a single discipline and collaboration across multiple disciplines within one office and multiple offices. Azhar (2011) emphasizes as we are leaving behind the requirement for BIM levels 1 and 2, the industry has overcome the obstacle of common data format and moving toward a shared product model. Nevertheless, the existing AEC collaboration model which works for documents but for model collaboration, has created a legacy mindset in the industry that needs to be replaced with "a new concept for virtual collaboration that promote new culture for collaborative working environment which will align model collaboration with a redefined industry collaboration protocols" empowered by BIM in cloud computing. The shared cloud-based product model should also provide multiple users with all-inclusive collaboration operations on model data simultaneously (Azhar, 2011). This will decrease the risk for conflicting information.

\section{BIM AND EDUCATION}

The construction industry has been struggling to maximize its productivity, quality and sustainability and, at the same time, minimize their wastes and loses. In a marketing research Forbes and Ahmed (2010) found that only 30\% working tie in the construction industry is effective and the rest is wasted (Forbes and Ahmed, 2010). Concerning that labor productivity level in the construction industry is almost the same as 100 years ago and yet globally $75 \%$ of construction work is not finished on time, the industry is looking for a digital revolution to speed up its productivity and bringing the cost down (E-Difice, 2017). 


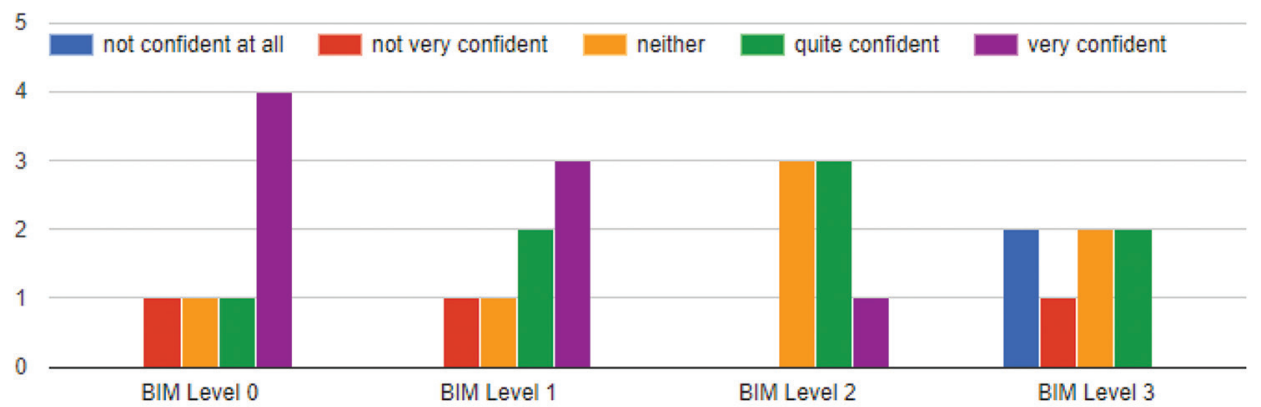

Fig. 5. Level of confidence in various building information modeling level among the professionals and academics responders to the survey of this study.

However, various market analyses and the study shows that building performance and operation are immensely improved by adopting BIM (Azhar, 2011), but when new technologies are adopted more work practices and learning needed to adjust to the new cultural environment. Therefore, moving beyond the software concepts, a whole new institutional workspace is created which means more intense collaboration between different disciplines that require fundamental changes in their way of working and acting.

Despite a common definition, we generally notice that BIM emerges differently to different people which are the source for failed collaboration culture. This is caused due to various pathway of BIM implementation, training, and engaging of workforce strategies across the AEC industry. However, with the current increase of design complexity globally, it is urgently required to bring closer the academic collaboration of the disciplines involved in the design and construction process (Poerschke, et al., 2010) for a common BIM ground to empower future graduates engaging in the industry.

Isolation of AEC students within discipline-specific education programs, departments, and schools has impacted on graduates' ability to function within interdisciplinary design teams when they enter the industry. Not only new graduates commonly hindered by poor cross-disciplinary communication, coordination, and negotiation skills but also they emerge from educational institutions with a narrow perception of what it means to participate in the design process as a member of their specific discipline. Understanding the goals and constraints of other disciplines is key to working well in cross-disciplinary projects. They have still not become a common setting since they are challenged with coordinating different learning objectives, curricula schedules, and teaching responsibilities, as well as different research and design cultures that exist among the disciplines (Fruchther et al., 2003).

Education is an essential part of successful BIM adoption, which is becoming widespread in the AEC professions, and it is increasingly expected from graduates to know this technology. Attempts to cultivate collaboration between departments such as architecture, architectural engineering, civil engineering, and other design disciplines have been ongoing for decades (Fruchter, 2003). Academia should not only react to this expectation but also should take the lead in researching the effects of BIM concerning, for example, the changes of collaboration structures, business models, and the design process. (Fruchter and Lewis, 2003).

However, introducing the integrated environment and the use of BIM within undergraduate AEC programs will lead to an intensive collaborative educational experience for students of the involved disciplines and to the mutual understanding of technical and social aspects of a collaborative design process which is an increasing prerequisite of graduate marketability.

The EUBIM (2016) Task Group which is leading the European efforts to common BIM strategies and digital construction industry devise that "in order to create capacity in the effective and consistent use of BIM, training providers and academia need to be equipped with a common definition of the target behaviors expected by the BIM program." Without a consistent definition of the required skills, it is likely that training providers and academia will not be able to develop a sufficient capacity of capable skilled professionals for futuristic construction industry.

In single isolated attempts, it would be unproductive and cost ineffective for the BIM action group to develop industrywide BIM training courses and training materials. Therefore, it is more desirable to develop a skills framework that layout feasible learning outcomes which industry and academia can then respond to by developing courses and materials with target requirements.

The obviously common understanding, common data exchange, common ways of working will create a foundation for consistent upskilling, training, and education that regulate the future market. This study conducted a survey among academics and students of AEC disciplines at several universities in the UK and the KRI to understand the current trends and understandings related to BIM in higher education. The survey received 280 responses in total, namely 241 from the UK and 39 responses from KRI academics.

Analysis of the respondents revealed that $86 \%$ of the respondents had a good level of understanding of BIM concept and value whereas only $43 \%$ had ever been engaged in a BIM-based project processing. However, over $90 \%$ of the respondents strongly believed in skill values gained through the BIM curriculum in graduates' marketability. Over $82 \%$ agreed that adopting new technologies in the construction industry 
is directly related to the graduate's relevant skills when they start their careers, therefore, they confirmed that the future trends are good reasons to adopt the new technologies in their curriculums (Fig. 6). Yet $85 \%$ of respondents confirmed that lack of deep knowledge, clarity and right skills of BIM have hindered the higher education to integrate the BIM in their programs, especially in more technical disciplines.

The overall knowledge about BIM level 3 and the concept of cloud computing appeared to be very low among academics and students. Nearly $87 \%$ of respondents said that they are not confident about BIM level 3 and they do not know what cloud computing means to implementation BIM level 3 or advance shared product model. They also see the lack of knowledge and clarity of BIM related problems as major indicators not providing BIM education at their universities (Fig. 7).
However, it is a huge gap between different level of performing BIM strategies in higher education and the AEC industry which does not show many changes despite the increasing e-resources in our daily life. "This is counterproductive to the overall strategic role and contributions of HEIs to the ongoing BIM digital revolution in AEC industry" (Khosrowshahi, et al., 2015). Furthermore, an earlier study by BAF in 2015 revealed that $40 \%$ of questioned academic institutes considered themselves not adequately informed on BIM and the UK Government implementation strategy of BIM levels.

Embedded BIM in undergraduate programs will have a direct impact on the program structure to enable the BIM model to empower learning but adopting this strategy still at a very slow pace (BFA, 2013).

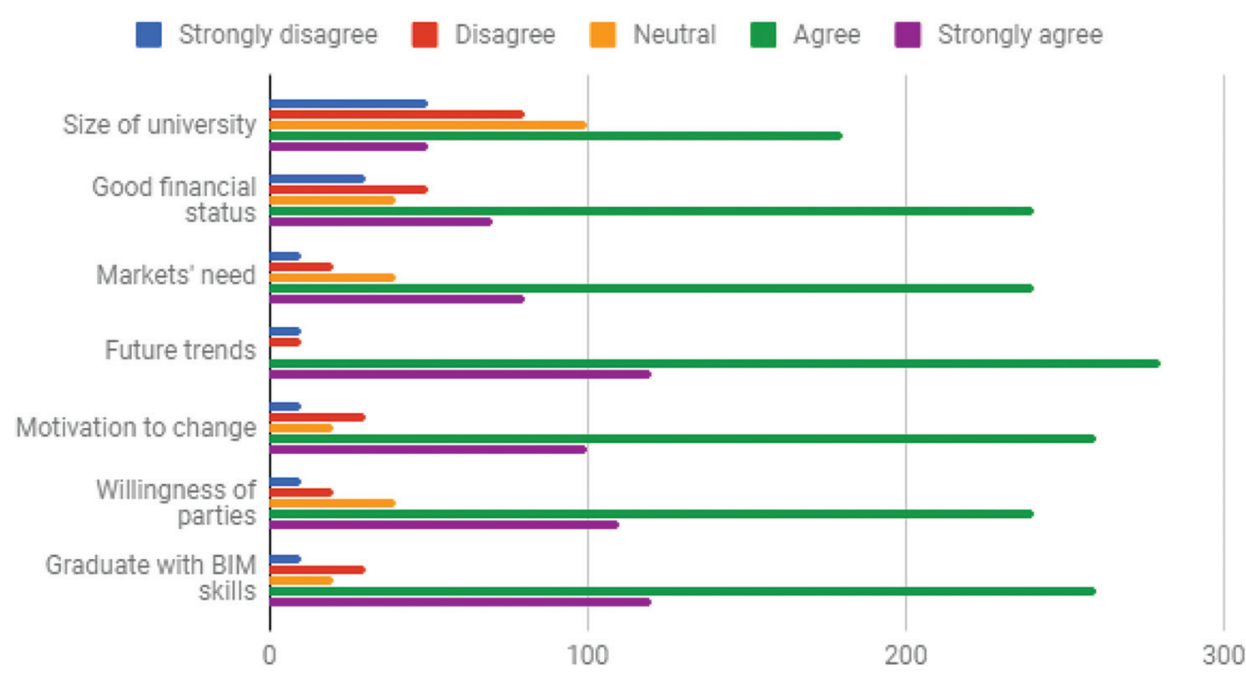

Fig. 6. Academics see the future trends a good reason to adopt new technology.

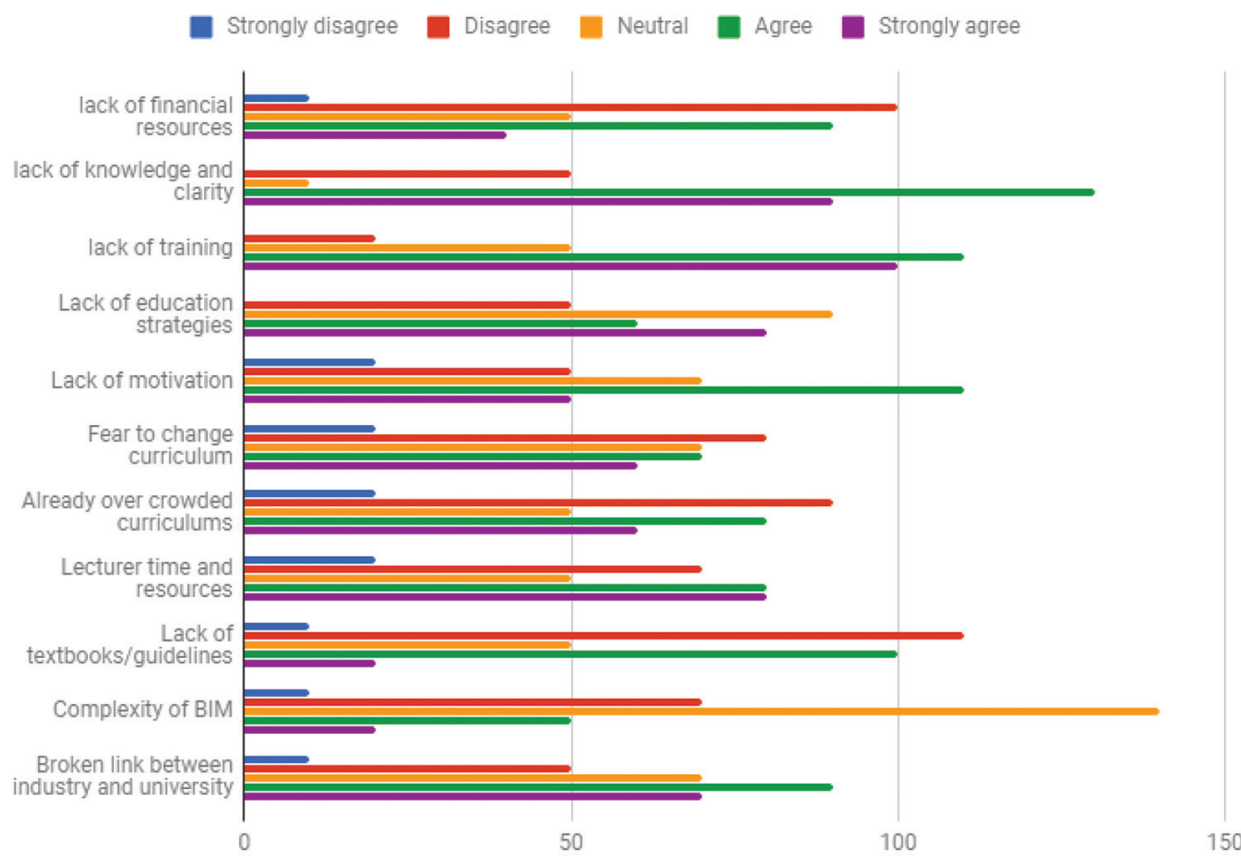

Fig. 7. Academic indicating lack of clarity and knowledge in building information modeling as major bottlenecks in providing building information modeling education. 
The BAF (2013) guidelines are providing expected learning outcomes on different levels of knowledge and understanding, practical skills, and transferable skills for embedding the BIM into undergraduate and postgraduate programs, but this has not been widely used by educational yet.

\section{RECOMMENDATIONS}

It is now widely acknowledged that the productivity and economic benefits of BIM to the AEC industry widely known and the technology to utilize BIM is rapidly advancing. Yet BIM adoption has been much slower than anticipated (Azhar, et al., 2008 and ARC 2015).

The EUBIM handbook put this in realistic figures and states "if the wider adoption of BIM across Europe delivered $10 \%$ savings to the construction sector then an additional $£ 115$ billion would be generated for the $£ 1.2$ trillion market. Even this impact could be small when compared with the potential social and environmental benefits that could be delivered to the climate change and resource efficiency agenda" (EUBIM, 2016).

BIM adoption would ultimately impose a change in the existing project processing and the whole culture of tradition AEC engagement. An integrated model development needs greater remotely collaboration and communication across disciplines that need to make sense of a virtual world. This means that a different approach to product model development is required in a virtual collaborative setting where all relevant factors contribute to a single shared model. This is a resolution for dynamic construction industry.

To make BIM more feasible for such a dynamic construction industry the shared collaborative environment need to facilitate variety organizational concepts to be selected that suit all standard data management, possible structural and project requirements and accommodate different business models to suit varied industry needs out of the package which is collected and shared from global project processing experiences.

However, these changes in the historical habits of the current industry require actors that have an educational and training background to understand that the culture of virtual working space collaboration in the sense of time and location. Such cultural education cannot be achieved through fragmented seminars but through programs of undergraduate degrees with marketing learning outcomes (Zhang et al., 2016).

In accordance with propagating the new culture of working, the industry needs to identify shortcomings and remove or redefine hurdles for the use of digital data in relation to liability, ownership, and rights that constrain benefits resulting from the wider adoption of BIM in a new ideal future working environment.

Hopkirk (2017) as well as this study reveals that currently there is BIM skills shortage among large companies in the UK. The survey indicated whereas large companies admitting the financial gain of using BIM but they cannot always find the skilled workforce they need (Hopkir, 2017). This clearly shows how the future architect and engineering should be educated with greater marketability in mind to speed up the industry adoption of BIM.
Nevertheless, the problems related to using BIM can be categorized into logistical and technical concerns. For the industry to move forward, the concept of ownership and intellectual property rights need to be changed. As the technical definition of product models has been fragmented to the smallest process and elements, then the ownership needs to be defined as one actors' specific action on these fragmented elements that are digitally signed rather than the whole product model. To facilitate such understanding and prevent disagreement over intellectual property problems, the best solution is to redefine the concept of ownership in a virtual working environment and educate actors with a new mindset for ownership rights and responsibilities that suit the future of the construction industry.

\section{CONCLUSIONS}

This study has evaluated the awareness of BIM in general and the problems surrounding the adoption process of BIM in AEC industry and the future AEC collaboration concept in particular. The study has identified the essence of the multidisciplinary collaborative working environment in an AEC virtual working space sharing a BIM-based product model and reflects a realistic capacity building for future construction industry professionals. Despite huge public and private investment in BIM related knowledge transfer, the adaptability pace has been slow. However, the success of a collaborative BIM-based project processing depends on group adoption by the actors that are expected to participate in the collaboration scenarios. Even though the NBS (2016) report revealed that $86 \%$ of respondents expect to be using BIM on at least some of their projects in 2016/2017, the working culture is far from the concept of single standard project processing. The future AEC engineers hold the key to making BIM the only method of processing a building project. Therefore, education delivery needs to evolve to accommodate all aspects of BIM processes throughout the product model lifecycle. In this approach, there is a clear need for a more rapid shift from historical habits of construction process practices to more desires futuristic BIM e-collaborative shared working processes.

The findings of this study provide useful information for AEC educational routes and industry considering adopting future level 3 BIM working strategy. The future of the construction industry is ever more defined by BIM advancement which is both exciting and challenging. It is obvious that BIM will enhance and redefine the collaboration as we know it and bring the AEC industry to much closer interdisciplinary actions and eventually lead to improved overall performance with a greater impact on the building lifecycle. This requires a closed-loop between AEC educational institutes, companies, and tools developer.

\section{ACKNOWLEDGMENT}

The author wishes to acknowledge his gratitude to the University of Nottingham, Department of Civil and Structural Engineering to facilitate this research work, 
and also special thanks to Koya University and Ministry of Higher Education in the KRI to approve the terms and conditions of this study.

\section{REFERENCES}

Aconex, 2017. BIM Management Software for Construction Projects. Available from: https://www.aconex.com/bim-management. [Last accessed on $2017 \mathrm{Aug}$ ].

ARC, 2015. Construction Professionals Make the Case for an Industry-Specific Cloud-based Document Management Application. ARC Document Solutions.

Autodesk, 2015. Getting More Value from your BIM Process with Autodesk Collaboration and Data Management Products. Available from: http:// www.static-dc.autodesk.net/content/dam/autodesk/www/solutions/buildinginformation-modeling/bim-value/bim-data-management-and-collaborationsept-2013.pdf. [Last accessed on 2016 May 03].

Azhar, S., 2011. Building information modeling (BIM): Trends, benefits, risks, and challenges for the AEC industry. Leadership and Management in Engineering, 11(3), pp.241-252.

Azhar, S., Hein, M. and Sketo, B., 2008. Building Information Modeling: Benefits, Risks and Challenges." Proc., $44^{\text {th }}$ Associated Schools of Construction National Conference, Auburn, AL.

Bazjanac, V., 2006. Information and Communication Technologies Improving Efficiencies. CRC, Boca Raton, FL.

Bhargav, D. and Lauri, K., 2009. Collaborative knowledge management a construction case study. Automation in Construction, 18(7), pp.894-902.

BIM Academic Forum UK., 2013. Embedding BIM within the Taught Curriculum. BS 1192, 2007, Collaborative Production of Architectural, Engineering and Construction Information Code of Practice, Annex (PAS 1192-2), BSI.

Dassault Systèmes, 2014. Architecture, Engineering and Construction Endto-End Collaboration by BIM Enabled by BIM Level 3, An Industry Approach Based on Best Practices from Manufacturing. Available from: http://www. emailing.3ds.com/res/dassault/a0dcaca10708f569ec7fb57ec7e577f5.pdf. [Last accessed on $2016 \mathrm{Feb}]$.

Eadie, R., Browne, M., Odeyinka, H., McKeown, C. and McNiff, S., 2015. A survey of current status of and perceived changes required for BIM adoption in the UK. Built Environment Project and Asset Management, 5(1), pp.4-21.

Eastman, C., Teicholz, P., Sacks, R. and Liston, K., 2008. BIM handbook: A Guide to Building Information Modeling for Owners, Managers, Designers, Engineers, and Contractors. $1^{\text {st }}$ ed. Publisher: Wiley, Hobokenpp, p. 490.

E-difice, 2017. The Construction Sector is in Need of a R-e-VOLUTION. Available from: http://www.e-difice.com/en. [Last accessed on $2017 \mathrm{Jul}]$.

EUBIM Task Group, 2016. Handbook for the Introduction of Building Information Modelling by the European Public Sector, The European Union. EUBIM Task Group, Europe.

Forbes, L.H. and Ahmed, S.M., (Eds.) 2010. Modern Construction: Lean Project Delivery and Integrated Practices (Industrial Innovation Series). CRC Press, Boca Raton, FL.

Fruchter, R. and Lewis, S., 2003. Mentoring models in support of P5BL in architecture/engineering/construction teamwork. International Journal of Engineering Education, 19(5), pp.663-671.

Fruchter, R., 2003. Innovation in Engaging Learning and Global Teamwork Experiences. In Information Technology 2003: Towards a Vision for Information Technology in Civil Engineering: Proceedings of the Fourth Join International Symposium on Information Technology in Civil Engineering, Nashville, Tennessee.

Fruchther, R. and Lewis, S., 2003. Mentoring models in support of p5bl in architecture/engineering/construction global teamwork. International Journal
Engineering Education, 19(5), pp.663-671.

Hardin, B., 2009. BIM and construction management. Wiley, Indianapolis, IN.

Hopkirk, E., 2017. Skills Shortage Hits BIM Uptake, Survey Finds, News in Building Design. Available from: https://www.bdonline.co.uk/news/skillsshortage-hits-bim-uptake-survey-finds/5090532.article. [Last accessed on 2017 Nov 14].

Howard, R. and Björk, B., 2008. Building information modelling experts' views on standardisation and industry deployment. Journal of Advanced Engineering Informatics Archive, 22(2), pp.271-280.

Kaner, I., Sacks, R., Kassian, W. and Quitt, T., 2008. Case studies of BIM adoption for precast concrete design by mid-sized structural engineering firms. ITcon, 13, pp.303-323.

Khanzode, A., Fischer, M. and Reed, D., 2008. Benefits and lessons learned of implementing building virtual design and construction (VDC) technologies for coordination of mechanical, electrical, and plumbing (MEP) systems on a large healthcare project. ITcon, 13, pp.324-342.

Khosrowshahi, F., Greenwood, D., Pittard, S. and Garvey, R., 2015. Current Position and Associated Challenges of BIM Education in UK Higher Education, BIM Academic Forum. Project Report.

Manning, R. and Messner, J., 2008. Case studies in BIM implementation for programming of healthcare facilities. ITcon, 13, pp.246-257.

Mills, F., 2015. Levels of Definition Explained. available form: https://www. theb1m.com/video/levels-of-definition-explained. [Last accessed on $2017 \mathrm{Sep}$ ].

NBS, 2014. National BIM Report Survey. Available from: https://www.thenbs. com/knowledge/nbs-national-bim-report-2014. [Last accessed on 2016 May].

NBS, 2016. National BIM Report Survey. Available from: https://www.thenbs. com/knowledge/national-bim-report-2016. [Last Accessed on 2016 Aug].

NBS-US, 2015. National BIM Standard-United States ${ }^{\circledR}$ Version 3. Available from: https://www.nationalbimstandard.org/files/NBIMS-US_V3_5.3_BIM_ PxP_Guide.pdf. [Last accessed on 2017 Aug 22].

Olofsson, T., Lee, G. and Eastman, C., 2008. Case studies of BIM in use. ITcon, 13, pp.244-245.

Poerschke, U., Holland, R.J., Messner, J.I. and Pihlak, M., 2010. BIM Collaboration Across Six Disciplines, Proceedings of the International Conference on Computing in Civil and Building Engineering, pp.575-671.

Roshani, D. and Tizani, W., 2005. Integrated IFC based Collaborative Building Design using Internet Technology. Civil-Comp Press, Stirlingshire, UK, pp.58.

Shafiq, M.T., Matthews, J. and Lockley, S.R., 2013. A study of BIM collaboration requirements and available features in existing model collaboration systems. Journal of Information Technology in Construction, 18, pp.148-161.

Shin, T.S., 2017. Building information modeling (BIM) collaboration from the structural engineering perspective, Korea. International Journal of Steel Structures, 17(1), pp.205-214.

Singh, V., Gu, N. and Wang, X., 2011. A theoretical framework of a BIM-based multi-disciplinary collaboration platform. Automation in Construction, 20, pp.134-144.

Stangeland, B.K., 2011. BIM Collaboration Format (BCF), Building SmartInternational Alliance for Interoperability. Available from: $<\mathrm{https}: / / \mathrm{www}$. web.archive.org/web/20120323221319>; http://www.iug.buildingsmart.com/ resources/abu-dhabi-iug-meeting/IDMC_017_1.pdf. [Last accessed on 2017 Jan 24].

Thomassen, M., 2011. BIM and Collaboration in the AEC Industry, A Dissertation at Aalborg University. Denmark.

Zhang, J., Schmidt, K. and Li, H., 2016. BIM and sustainability education: Incorporating instructional needs into curriculum planning in CEM programs accredited by ACCE. Sustainability (Switzerland), 8(6), p.525. 